\title{
Approximate Solution of Linear Fuzzy Initial Value Problems Using Modified Variaional Iteration Method
}

\author{
Hussein M. Sagban and Fadhel S. Fadhel* \\ Department of Mathematics and Computer Applications, College of Science, Al-Nahrain University, \\ Baghdad-Iraq
}

\begin{tabular}{|c|c|}
\hline Article's Information & Abstract \\
\hline $\begin{array}{l}\text { Received: } \\
\text { 03-10-2021 } \\
\text { Accepted: } \\
\text { 14-11-2021 } \\
\text { Published: } \\
\text { 31-12-2021 }\end{array}$ & \multirow[t]{2}{*}{$\begin{array}{l}\text { The main objective of this paper is to solve fuzzy initial value problems, in which } \\
\text { the fuzziness occurs in the initial conditions. The proposed approach, namely the } \\
\text { modified variational iteration method, will be used to find the solution of fuzzy } \\
\text { initial value problem approximately and to increase the rate of convergence of the } \\
\text { variational iteration method. From the obtained results, as it is expected, the } \\
\text { approximate results of the proposed method are more accurate than those results } \\
\text { obtained without using the modified variational iteration method. }\end{array}$} \\
\hline $\begin{array}{l}\text { Keywords: } \\
\text { Modified variational iteration } \\
\text { method } \\
\text { Fuzzy initial value problem } \\
\text { Triangular fuzzy number } \\
\text { Ordinary differential equation } \\
\text { General Lagrange multiplier }\end{array}$ & \\
\hline $\begin{array}{l}\text { DOI: } 10.22401 / \text { ANJS.24.4.05 } \\
{ }^{*} \text { Corresponding author: fadhel.s }\end{array}$ & nahrainuniv.edu.eq \\
\hline
\end{tabular}

\section{Introduction}

Fuzzy sets and their basic concepts were first introduced in 1965 by L. A. Zadeh in his seminal paper "Fuzzy sets" published in the journal Information and Control. Fuzzy logic as a tool is powerful for modeling uncertainty and processing vague notions, which are subjective information in mathematical models in which the main direction of development has been diverse and has application in solving many real problems, [1].

Rapid development of fuzzy differential equations in recent years as it is introduced by Abbasbandy S. in 2002 [2] for solving fuzzy initial value problems (FIVP's) using Taylor's method, which is discussed in details and followed completely by error analysis. Jafari H. in 2012 in his article solved the $n$-th order linear and nonlinear fuzzy ordinary differential equations (FODE's) with fuzzy initial conditions by using variational iteration method (VIM), [3]. Subsequently, Allahvivallco T. et al. in 2013 [4] solves fuzzy differential equations which are nonlinear by using the VIM and thereafter in 2014 Janeel A. F. used the VIM to approximate the analytical solution for an initial value problem involving fuzzy Duffing equation, [5]. Linear fuzzy ordinary differential equations of the $n$-th order were solved in 2013 at the same time by Xianbin Guo and Deauan Shang [6] and find the approximate solution in which the sign of the coefficient functions are maintained and investigated by the method of undetermined fuzzy coefficients. In 2016, Ghazenfar B. gave the numerical solution of hybrid fuzzy differential equations by applying also the variational iteration method, [7].

Adomian decomposition method have been applied and illustrated by Navayanan in 2017 for solving nonlinear Cauchy problem, in which the convergence analysis of this problem is also investigated in this article, [8]. Solution of delay differential equations with fuzzy domain using the VIM based He's polynomials were introduced in 2017 by Narayanamoorthy S. and Yookesh T. L., [9] Behzadi Wh. S. in 2018 proposed the fuzzy VIM to solve $2^{\text {nd }}$-order AbelVolterra fuzzy intrgo-differential equation, [10].

In this paper, in order to improve the accuracy of the results and the rate of convergence of the approximate numerical results to the exact solution, we will apply the modified VIM to solve $n$-th order FIVP's of the form:

$$
\tilde{y}^{(n)}(x)=f\left(x, \tilde{y}(x), \tilde{y}^{\prime}(x), \ldots, \tilde{y}^{(n-1)}(x)\right), x \in[0,1]
$$

with initial conditions given according to triangular fuzzy numbers:

$$
\tilde{y}(0)=\tilde{b}_{0}, \tilde{y}^{\prime}(0)=\tilde{b}_{1}, \ldots, \tilde{y}^{(n-1)}(x)=\tilde{b}_{n-1}
$$

where $x$ is the state variable, $\tilde{y}$ is an unknown fuzzy function which is depend on $x, \tilde{b_{0}}, \tilde{b_{1}}, \ldots, \tilde{b_{n-1}}$ are triangular fuzzy numbers and $f$ is any given function satisfying the conditions of the existence and uniqueness theorem of FIVP's. 


\section{Al-Nahrain Journal of Science}

ANJS, Vol.24 (4), December, 2021, pp. 32-39

\section{Basic Concepts}

To make this paper of self-contents, as possible, some fundamental concepts and basic definitions related to this work will be given for completeness purpose and those more primitive concepts will not be given.

Definition 1, [11]. A triangular fuzzy number is a mapping $u: \mathbb{R} \longrightarrow[0,1]$, which satisfies:

1. $u$ is upper semi-continuous.

2. There exists real numbers $a, b$ and $c$, such that $a \leq b \leq c$ and

1. $u(x)=0$ outside some interval $[a, c]$.

2. $u(x)$ is monotonic increasing on $[a, b]$.

3. $u(x)$ is monotonic decreasing on $[b, c]$.

4. $u(x)=1$ at $x=b$.

Definition 2, [12]. The set of all elements that belong to the fuzzy set $\tilde{u}$ at least to the degree $\alpha$ is called the $\alpha$-level set $u_{\alpha}=\left\{x \in X: \mu_{\widetilde{u}}(x) \geq \alpha\right\}$

where $\mu_{\widetilde{u}}$ refers to the characteristic function related to the fuzzy set $\tilde{u}$.

Fuzzy numbers may be characterized or parameterized in terms of $\alpha$-level sets as it is given in the next definition:

Definition 3, [11]. A fuzzy number $u$ in parametric form is a pair $(\underline{u}(\alpha), \bar{u}(\alpha)), 0 \leq \alpha \leq 1$ which satisfy the following requirements:

1. $\underline{u}(\alpha)$ is a bounded monotonic increasing right continuous function.

2. $\bar{u}(\alpha)$ is a bounded monotonic decreasing left continuous function.

3. $\underline{u}(\alpha) \leq \bar{u}(\alpha)$, for all $0 \leq \alpha \leq 1$.

Remark 1, [12]. For an arbitrary two fuzzy numbers $\tilde{u}=(\underline{u}(\alpha), \bar{u}(\alpha))$ and $\tilde{v}=(\underline{v}(\alpha), \bar{v}(\alpha))$, the following algebraic operations may be fulfilled:

1. If $k$ is any real number, then:

$$
k \tilde{u}=\left\{\begin{array}{l}
(k \underline{u}(\alpha), k \bar{u}(\alpha)), \text { if } k \geq 0 \\
(k \bar{u}(\alpha), k \underline{u}(\alpha)), \text { if } \quad k<0
\end{array}\right.
$$

2. $\tilde{u} \mp \tilde{v}=(\underline{u}(\alpha) \mp \underline{v}(\alpha), \bar{u}(\alpha) \mp \bar{v}(\alpha))$.

3. $\tilde{u} \tilde{v}=(\min s, \max s)$, where:

$s=\{\underline{u} \underline{v}, \underline{u} \bar{v}, \bar{u} \underline{v}, \bar{u} \bar{v}\}$

For setting the problem formulation, the general form of the $n$-th order FODE with constant coefficients related to equation (1) takes the form $[13,14]$ :

$$
\begin{aligned}
& \tilde{y}^{(n)}(x)+c_{n-1} \tilde{y}^{(n-1)}(x)+\ldots+c_{1} \tilde{y}^{\prime}(x)+ \\
& c_{0} \tilde{y}(x)=\tilde{R}(x), x \geq 0
\end{aligned}
$$

with initial conditions given according to triangular fuzzy numbers:

$$
\tilde{y}(0)=\tilde{b_{0}}, \tilde{y}^{\prime}(0)=\tilde{b_{1}}, \ldots, \tilde{y}^{(n-1)}(x)=\tilde{b}_{n-1}
$$

where $c_{i}$ 's are real constants for all $i=0,1, \ldots, n-1$ and $\tilde{b_{0}}, \tilde{b_{1}}, \ldots, \tilde{b}_{n-1}$ are triangular fuzzy numbers. Here, $\tilde{y}$ is the solution to be determined as a fuzzy function.

Another representation of equation (1) may be given in terms of lower and upper solutions related to the r-levels of the fuzzy solution function. This is given in the next equation:

$$
\begin{aligned}
& {\left[\underline{y}^{(n)}(x, \alpha), \bar{y}^{(n)}(x, \alpha)\right]+} \\
& c_{n-1}\left[\underline{y}^{(n-1)}(x, \alpha), \bar{y}^{(n-1)}(x, \alpha)\right]+\ldots+ \\
& c_{1}\left[\underline{y}^{\prime}(x, \alpha), \bar{y}^{\prime}(x, \alpha)\right]+c_{0}[\underline{y}(x, \alpha), \bar{y}(x, \alpha)]= \\
& {[\underline{R}(x, \alpha), \bar{R}(x, \alpha)]}
\end{aligned}
$$

From operation (1) of Remark 1, three cases may be arises in solving the fuzzy differential equation, these cases may be summarized as follows:

Case 1: All the coefficients $c_{n-1}, c_{n-2}, \ldots, c_{0}$ are positive and hence equation (3) will be decomposed into the following nonfuzzy or crisp ordinary differential equations:

$$
\begin{aligned}
& \underline{y}^{(n)}(x, \alpha)+c_{n-1} \underline{y}^{(n-1)}(x, \alpha)+\ldots+c_{1} \underline{y}^{\prime}(x, \alpha)+ \\
& c_{0} \underline{y}(x, \alpha)=\underline{R}(x, \alpha)
\end{aligned}
$$

with initial conditions:

$$
\begin{aligned}
& \underline{y}(0, \alpha)=\underline{b}_{0}, \underline{y}^{\prime}(0, \alpha)=\underline{b}_{1}, \ldots, \underline{y}^{(n-1)}(0, \alpha)=\underline{b}_{n-1} \\
& \bar{y}^{(n)}(x, \alpha)+c_{n-1} \bar{y}^{(n-1)}(x, \alpha)+\ldots+c_{1} \bar{y}^{\prime}(x, \alpha)+ \\
& c_{0} \bar{y}(x, \alpha)=\bar{R}(x, \alpha)
\end{aligned}
$$
and

with initial conditions:

$$
\bar{y}(0, \alpha)=\bar{b}_{0}, \bar{y}^{\prime}(0, \alpha)=\bar{b}_{1}, \ldots, \bar{y}^{(n-1)}(0, \alpha)=\bar{b}_{n-1}
$$

Case 2: The coefficients $c_{n-1}, c_{n-2}, \ldots, c_{n-m}$ are positive and $c_{n-m-1}, c_{n-m-2}, \ldots, c_{1}, \mathrm{c}_{0}$ are negative and also equation (3) will be decomposed into the following crisp ordinary differential equations:

$$
\begin{aligned}
& \underline{y}^{(n)}(x, \alpha)+c_{n-1} \underline{y}^{(n-1)}(x, \alpha)+\ldots+ \\
& c_{n-m} \underline{y}^{(n-m)}(x, \alpha)+c_{n-m-1} \bar{y}^{(n-m-1)}(x, \alpha)+ \\
& c_{n-m-2} \bar{y}^{(n-m-2)}(x, \alpha)+\ldots+c_{1} \bar{y}^{\prime}(x, \alpha)+ \\
& c_{0} \bar{y}(x, \alpha)=\underline{R}(x, \alpha)
\end{aligned}
$$

with initial conditions:

$$
\begin{aligned}
& \underline{y}^{(n-1)}(0, \alpha)=\underline{b}_{n-1}, \ldots, \underline{y}^{(n-m)}(0, \alpha)=\underline{b}_{n-m}, \\
& \bar{y}^{(n-m-1)}(0, \alpha)=\bar{b}_{n-m-1}, \ldots, \bar{y}^{\prime}(0, \alpha)=\overline{b_{1}}, \\
& \bar{y}(0, \alpha)=\bar{b}_{0}
\end{aligned}
$$

and for the upper crisp solution:

$$
\begin{aligned}
& \bar{y}^{(n)}(x, \alpha)+c_{n-1} \bar{y}^{(n-1)}(x, \alpha)+\ldots+ \\
& c_{n-m} \bar{y}^{(n-m)}(x, \alpha)+c_{n-m-1} \underline{y}^{(n-m-1)}(x, \alpha)+ \\
& c_{n-m-2} \underline{y}^{(n-m-2)}(x, \alpha)+\ldots+c_{1} \bar{y}^{\prime}(x, \alpha)+ \\
& c_{0} \bar{y}(x, \alpha)=\bar{R}(x, \alpha)
\end{aligned}
$$




\section{Al-Nahrain Journal of Science}

ANJS, Vol.24 (4), December, 2021, pp. 32-39

$$
\begin{aligned}
& \bar{y}^{(n-1)}(0, \alpha)=\bar{b}_{n-1}, \ldots, \bar{y}^{(n-m)}(0, \alpha)=\bar{b}_{n-m}, \\
& \underline{y}^{(n-m-1)}(0, \alpha)=\underline{b}_{n-m-1}, \ldots, \underline{y}^{\prime}(0,, r)=\underline{b}_{1}, \\
& \underline{y}(0, \alpha)=\underline{b}_{0}
\end{aligned}
$$

Case 3: All the coefficients $c_{n-1}, c_{n-2}, \ldots, c_{0}$ are negative, then equation (3) will be decomposed into the following two initial value problems:

$$
\begin{aligned}
& \underline{y}^{(n)}(x, \alpha)+c_{n-1} \bar{y}^{(n-1)}(x, \alpha)+\ldots+c_{1} \bar{y}^{\prime}(x, \alpha)+ \\
& c_{0} \bar{y}(x, \alpha)=\underline{R}(x, \alpha)
\end{aligned}
$$

with initial conditions:

$$
\bar{y}(0, r)=\bar{b}_{0}, \bar{y}^{\prime}(0, r)=\bar{b}_{1}, \ldots, \bar{y}^{(n-1)}(0, r)=\bar{b}_{n-1}
$$

and

$$
\begin{aligned}
& \bar{y}^{(n)}(x, \alpha)+c_{n-1} \underline{y}^{(n-1)}(x, \alpha)+\ldots+c_{1} \underline{y}^{\prime}(x, \alpha)+ \\
& c_{0} \underline{y}(x, \alpha)=\bar{R}(x, \alpha)
\end{aligned}
$$

with initial conditions:

$$
\underline{y}(0, \alpha)=\underline{b}_{0}, \underline{y}^{\prime}(0, \alpha)=\underline{b}_{1}, \ldots, \underline{y}^{(n-1)}(0, \alpha)=\underline{b}_{n-1}
$$

In the next section, we will present a strict approach for solving a kind of equations using the VIM, which has not taken in consideration by H. Jafari et al. in their article [3]. They did not tap on the effect of the coefficients in the linear FIVP to be either positive or negative or mixed of which has its affection on the lower and upper solutions of the FIVP. Their results contradict the basic theory of FIVP with triangular fuzzy numbers, which is the equality of the lower and upper solutions at discretization level equals 1 .

\section{The Modified Variational Iteration Method}

In this section, we will use the VIM and its modification to solve FIVP's, but first the basic elements of the VIM and its modification for solving nonlinear operator equations will be introduced. For this purpose, consider the nonlinear equation:

$$
L \tilde{y}(x)+N \tilde{y}(x)=g(x)
$$

subject to certain initial conditions, where $L$ is a linear operator, $N$ is a nonlinear operator and $g$ is the nonhomogeneous term.

He's VIM may be boils down to the modification of the general Lagrange multiplier method so one may write equation (10) in the form:

$$
\begin{gathered}
\tilde{y}_{n+1}(x)=\tilde{y}_{n}(x)+\int_{0}^{x} \lambda(x, s)\left(L \tilde{y}_{n}(s)+N \tilde{y}_{n}^{*}(s)-\right. \\
g(s)) \mathrm{d} s
\end{gathered}
$$

where $\tilde{y}_{n}^{*}$ is considered here as a restricted variation, i.e., the first variation $\delta \tilde{y}_{n}^{*}=0$ and $\lambda$ is the general Lagrange multiplier which is identified optimally through variational theory and it is proved in some literatures (see for example $[15,16])$ to be of the following form for the $n$-th order ordinary differential equation:

$$
\lambda(x, s)=(-1)^{n} \frac{(s-x)^{n-1}}{(n-1) !}
$$

where $n \in \mathbb{N}$. So, the iteration equation (11) of the solution after substituting the general Lagrange multiplier (12) with the existence of the initial condition $\tilde{y}_{0}$ related to the nonlinear operator equation (10) will take the form:

$$
\begin{gathered}
\tilde{y}_{n+1}(x)=\tilde{y}_{n}(x)+\int_{0}^{x}(-1)^{n} \frac{(s-x)^{n-1}}{(n-1) !}\left(L \tilde{y}_{n}(s)+\right. \\
\left.N \tilde{y}_{n}(s)-g(s)\right) \mathrm{d} s
\end{gathered}
$$

Now, to modify and improve the accuracy of the approximate numerical results obtained by using the VIM for solving FODE's subject to certain initial conditions in terms of fuzzy numbers, as well as, increase the rate of convergence of the sequence of approximate numerical solutions to the exact solution. This development may be accomplished by adding and subtracting a suitable linear operator, say $L_{1}$, to the original operator equation (10) to get a new operator equation. This has a result of evaluating new general Lagrange multipliers, which will give the desired goal. Thus, the new modified operator has the form:

$$
L \tilde{y}(x)+L_{1} \tilde{y}(x)-L_{1} \tilde{y}(x)+N \tilde{y}(x)=g(x)
$$

or

$$
L^{*} \tilde{y}(x)+N^{*} \tilde{y}(x)=g(x)
$$

where:

$$
\begin{aligned}
& L^{*} \tilde{y}(x)=L \tilde{y}(x)+L_{1} \tilde{y}(x) \\
& N^{*} \tilde{y}(x)=N \tilde{y}(x)-L_{1} \tilde{y}(x)
\end{aligned}
$$

Hence, the correction functional may be constructed based on the new linear operator $L^{*}$ and thus the correction functional (2) will be:

$$
\begin{aligned}
\tilde{y}_{n+1}(x)= & \tilde{y}_{n}(x)+\int_{0}^{x} \lambda(x, s)\left[L \tilde{y}_{n}(s)+L_{1} \tilde{y}_{n}(s)-\right. \\
& \left.L_{1} \tilde{y}_{n}(s)+N \tilde{y}_{n}(s)-g(s)\right] \mathrm{d} s \\
= & \tilde{y}_{n}(x)+\int_{0}^{x} \lambda(x, s)\left[L^{*} \tilde{y}_{n}(s)+N^{*} \tilde{y}_{n}(s)-\right. \\
& g(s)] \mathrm{d} s
\end{aligned}
$$

and therefore, the value of the new general Lagrange multiplier will depend on the choice of the auxiliary linear operator $L_{1}$.

Now, since any $n$-th order FODE, linear or nonlinear, may be written in the following $n$-th order fuzzy initial value problem:

$$
\tilde{y}^{(n)}(x)=f\left(x, \tilde{y}(x), \tilde{y}^{\prime}(x), \ldots, \tilde{y}^{(n-1)}(x)\right), x \in[0,1]
$$

with initial conditions given as triangular fuzzy numbers:

$$
\tilde{y}^{(i)}(0)=\tilde{y}_{0}^{i}, i=0,1, \ldots, n-1
$$

Hence, the correction functional for equation (2) will be read for the lower and upper solutions as: 


\section{Al-Nahrain Journal of Science}

ANJS, Vol.24 (4), December, 2021, pp. 32-39

$$
\begin{aligned}
\underline{y}_{n+1}(x, \alpha)= & \underline{y}_{n}(x, \alpha)+\int_{0}^{x} \lambda(x, s)\left[\frac{d^{n}}{d s^{n}} \underline{y}_{n}(s, \alpha)+\right. \\
& L_{1} \underline{y}_{n}(s, \alpha)-L_{1} \underline{y}_{n}(s, \alpha)+f\left(s, \underline{y}_{n}(s, \alpha),\right. \\
& \left.\left.\underline{y}_{n}^{\prime}(s, \alpha), \ldots, \underline{y}_{n}^{(n-1)}(s, \alpha)\right)\right] \mathrm{d} s \ldots(16) \\
\bar{y}_{n+1}(x, \alpha)= & \bar{y}_{n}(x, \alpha)+\int_{0}^{x} \lambda(x, s)\left[\frac{d^{n}}{d s^{n}} \bar{y}_{n}(s, \alpha)+\right. \\
& L_{1} \bar{y}_{n}(s, \alpha)-L_{1} \bar{y}_{n}(s, \alpha)+f\left(s, \bar{y}_{n}(s, \alpha),\right. \\
& \left.\left.\bar{y}_{n}^{\prime}(s, \alpha), \ldots, \bar{y}_{n}^{(n-1)}(s, \alpha)\right)\right] \mathrm{d} s \quad \ldots(17)
\end{aligned}
$$

where $r \in[0,1]$.

\section{Application of the VIM and Numerical \\ Simulation}

Two examples will be considered in this section as an application to the proposed approach in which their solutions are carried out using Mathcad 15 computer software.

Example 1. Consider the second order linear FODE:

$$
\tilde{y}^{\prime \prime}(x)+\tilde{y}(x)=-x, x \in[0,1]
$$

with initial conditions for all $\alpha \in[0,1]$ :

$$
\begin{aligned}
& \tilde{y}(0, \alpha)=(0.1 \alpha-0.1,0.1-0.1 \alpha) \\
& \tilde{y}^{\prime}(0, \alpha)=(0.088+0.1 \alpha, 0.28-0.1 \alpha)
\end{aligned}
$$

For comparison purpose, the exact solution is given in [3] by:

$\underline{Y}_{-}(x, \alpha)=(0.1 \alpha-0.1) \cos (x)+(1.088+0.1 \alpha) \sin (x)-x$

$\bar{Y}(x, \alpha)=(0.1-0.1 \alpha) \cos (x)+(1.288-0.1 \alpha) \sin (x)-x$

This example is also solved in [3] using the VIM, in which they got the results after three iterations and their absolute error with exact solution are given in Table 1:

Table 1. The absolute error between the exact and approximate solutions $y$ and $\bar{y}$ of Example 1 using the VIM.

\begin{tabular}{|c|c|}
\hline$\left|\underline{Y}-\underline{y}_{3}\right|$ & $\left|\bar{Y}-\bar{y}_{3}\right|$ \\
\hline $5 \times 10^{-7}$ & $2.14 \times 10^{-7}$ \\
\hline $5 \times 10^{-7}$ & $2.13 \times 10^{-7}$ \\
\hline $5 \times 10^{-7}$ & $2.113 \times 10^{-7}$ \\
\hline $5 \times 10^{-7}$ & $2.096 \times 10^{-7}$ \\
\hline $5 \times 10^{-7}$ & $2.08 \times 10^{-7}$ \\
\hline $5 \times 10^{-7}$ & $2.013 \times 10^{-7}$ \\
\hline $5 \times 10^{-7}$ & $2.046 \times 10^{-7}$ \\
\hline $5 \times 10^{-7}$ & $2.03 \times 10^{-7}$ \\
\hline $5 \times 10^{-7}$ & $2.013 \times 10^{-7}$ \\
\hline $5 \times 10^{-7}$ & $1.997 \times 10^{-7}$ \\
\hline $5 \times 10^{-7}$ & $1.98 \times 10^{-7}$ \\
\hline
\end{tabular}

Equation (18) may be rewritten using the proposed approach after adding and subtracting the linear operator $\tilde{y}^{\prime}(x)$ as:

$$
\tilde{y}^{\prime \prime}(x)+\tilde{y}^{\prime}(x)-\tilde{y}^{\prime}(x)+\tilde{y}(x)=-x, x \in[0,1]
$$

In comparison with equation (14), let the linear operator $L^{*} \tilde{y}(x)=\tilde{y}^{\prime \prime}(x)+\tilde{y}^{\prime}(x)$ and the nonlinear operator $N^{*} \tilde{y}(x)=\tilde{y}(x)-\tilde{y}^{\prime}(x)$ and thus applying the VIM implies to:

$$
\begin{aligned}
\tilde{y}_{n+1}(x)= & \tilde{y}_{n}(x)+\int_{0}^{x} \tilde{\lambda}(x, s)\left[L^{*} \tilde{y}_{n}(s)+N^{*} \tilde{y}_{n}(s)-\right. \\
& g(s)] \mathrm{d} s \\
= & \tilde{y}_{n}(x)+\int_{0}^{x} \tilde{\lambda}(x, s)\left[\tilde{y}_{n}^{\prime \prime}(s)+\tilde{y}_{n}^{\prime}(s)+\right. \\
& \left.\tilde{y}_{n}(s)-\tilde{y}_{n}^{\prime}(s)+s\right] \mathrm{d} s
\end{aligned}
$$

Taking the first variation with respect to $\tilde{y}_{n}$ and noting that $\delta N^{*} \tilde{y}_{n}(s)=0$, then:

$$
\begin{gathered}
\tilde{y}_{n+1}(x)=\delta \tilde{y}_{n}(x)+\delta \int_{0}^{x} \tilde{\lambda}(x, s)\left[\tilde{y}_{n}^{\prime \prime}(s)+\tilde{y}_{n}^{\prime}(s)+\right. \\
\left.\tilde{y}_{n}(s)-\tilde{y}_{n}^{\prime}(s)+s\right] \mathrm{d} s
\end{gathered}
$$

Thus, we obtain the Euler-Lagrange equation:

$$
\tilde{\lambda}^{\prime \prime}(x, s)+\tilde{\lambda}^{\prime}(x, s)=0
$$

and the natural boundary conditions:

$$
1-\left.\tilde{\lambda}^{\prime}(x, s)\right|_{s=x}=0,\left.\tilde{\lambda}(x, s)\right|_{s=t} x=0
$$

We therefore identify the Lagrange multiplier for the lower and upper cases in the form:

$$
\underline{\lambda}(x, s)=\bar{\lambda}(x, s)=e^{s-x}-1
$$

and thus the lower and upper approximate solutions for all $r \in[0,1]$ and $n=0,1, \ldots$

$$
\begin{aligned}
\underline{y}_{n+1}(x, \alpha)= & \underline{y}_{n}(x, \alpha)+\int_{0}^{x}\left(e^{s-x}-1\right)\left[\underline{y}_{n}^{\prime \prime}(s, \alpha)+\right. \\
& \left.\underline{y}_{n}(s, \alpha)+s\right] \mathrm{d} s \\
\bar{y}_{n+1}(x, \alpha)= & \bar{y}_{n}(x, \alpha)+\int_{0}^{x}\left(e^{s-x}-1\right)\left[\bar{y}_{n}^{\prime \prime}(s, \alpha)+\right. \\
& \left.\bar{y}_{n}(s, \alpha)+s\right] \mathrm{d} s
\end{aligned}
$$

Choosing:

$$
\begin{aligned}
& \underline{y}_{0}(x, \alpha)=(0.1 \alpha-0.1)-(0.088+0.1 \alpha) x \\
& \bar{y}_{0}(x, \alpha)=(0.1-0.1 \alpha)-(0.28-0.1 \alpha) x
\end{aligned}
$$

We obtain the first lower and upper approximate solutions $y_{1}(x, \alpha)=(0.1 \alpha-0.1)-(0.088+0.1 \alpha) x+(0.1 \alpha-0.1)$

$$
\begin{aligned}
& +(1.088+0.1 \alpha)(x-1)-(0.1 \alpha-0.1) x- \\
& (0.088+0.1 \alpha) \frac{x^{2}}{2}-\frac{x^{2}}{2}-(0.1 \alpha-0.1) e^{-x}+ \\
& (1.088+0.1 \alpha) e^{-x}
\end{aligned}
$$




\section{Al-Nahrain Journal of Science}

ANJS, Vol.24 (4), December, 2021, pp. 32-39

$$
\begin{aligned}
\bar{y}_{1}(x, \alpha)= & (0.1-0.1 \alpha)+(0.288-0.1 \alpha) x+(0.1-0.1 \alpha) \\
& +(1.288-0.1 \alpha)(x-1)-(0.1-0.1 \alpha) x- \\
& (0.288-0.1 \alpha) \frac{x^{2}}{2}-\frac{x^{2}}{2}-(0.1-0.1 \alpha) e^{-x}+ \\
& (1.288-0.1 \alpha) e^{-x}
\end{aligned}
$$

Comparison using the absolute error between the approximate solution with the exact solutions, for the lower and upper cases, are given in Table 2.

Table 2. The absolute error between the exact and approximate solutions $\underline{y}$ and $\bar{y}$ of Example 1 using the new VIM.

\begin{tabular}{|c|c|}
\hline $\mid \underline{\underline{Y}-\underline{y}_{1} \mid}$ & $\left|\bar{Y}-\bar{y}_{1}\right|$ \\
\hline \hline $1.613 \times 10^{-8}$ & $1.712 \times 10^{-8}$ \\
\hline $1.447 \times 10^{-8}$ & $1.546 \times 10^{-8}$ \\
\hline $1.281 \times 10^{-8}$ & $1.379 \times 10^{-8}$ \\
\hline $1.114 \times 10^{-8}$ & $1.213 \times 10^{-8}$ \\
\hline $9.482 \times 10^{-9}$ & $1.047 \times 10^{-8}$ \\
\hline $7.819 \times 10^{-9}$ & $8.805 \times 10^{-9}$ \\
\hline $6.157 \times 10^{-9}$ & $7.143 \times 10^{-9}$ \\
\hline $4.494 \times 10^{-9}$ & $5.480 \times 10^{-9}$ \\
\hline $2.832 \times 10^{-9}$ & $3.818 \times 10^{-9}$ \\
\hline $1.169 \times 10^{-9}$ & $2.156 \times 10^{-9}$ \\
\hline $4.980 \times 10^{-10}$ & $4.980 \times 10^{-10}$ \\
\hline
\end{tabular}

Example 2. Consider the second order linear FODE:

$$
\tilde{y}^{\prime \prime}(x)-4 \tilde{y}^{\prime}(x)+4 \tilde{y}(x)=0, x \in[0,1]
$$

with initial conditions for all $\alpha \in[0,1]$ :

$$
\tilde{y}(0, \alpha)=(2+\alpha, 4-\alpha), \tilde{y}^{\prime}(0, \alpha)=(5+\alpha, 7-\alpha)
$$

For comparison purpose, the exact solution is given in [3] by:

$$
\begin{aligned}
& \underline{Y}_{-}(x, \alpha)=(2+\alpha) e^{2 x}+(1-\alpha) x e^{2 x} \\
& \bar{Y}(x, \alpha)=(4-\alpha) e^{2 x}+(\alpha-1) x e^{2 x}
\end{aligned}
$$

Now, by taking $L_{1} \tilde{y}(x)=\tilde{y}^{\prime}(x)$, then equation (19) may be written as:

$$
\tilde{y}^{\prime \prime}(x)-4 \tilde{y}^{\prime}(x)+4 \tilde{y}(x)+\tilde{y}^{\prime}(x)-\tilde{y}^{\prime}(x)=0
$$

and so:

$$
\tilde{y}^{\prime \prime}(x)+\tilde{y}^{\prime}(x)+4 \tilde{y}(x)-5 \tilde{y}^{\prime}(x)=0
$$

Therefore, according to the proposed approach, by letting $L^{*} \tilde{y}(x)=\tilde{y}^{\prime \prime}(x)+\tilde{y}^{\prime}(x)$ and assume virtually the nonlinear operator $N^{*} \tilde{y}(x)=4 \tilde{y}(x)-5 \tilde{y}^{\prime}(x)$, then applying the VIM implies to:

$$
\begin{aligned}
\tilde{y}_{n+1}(x)= & \tilde{y}_{n}(x)+\int_{0}^{x} \tilde{\lambda}(x, s)\left[\tilde{y}_{n}^{\prime \prime}(s)+\tilde{y}_{n}^{\prime}(s)+4 \tilde{y}_{n}^{\prime *}(s)\right. \\
& \left.-5 \tilde{y}_{n}^{*}(s)\right] \mathrm{d} s
\end{aligned}
$$

Similarly, as in Example 1, we have the Lagrange multiplier:

$$
\underline{\lambda}(x, s)=\bar{\lambda}(x, s)=e^{s-x}-1
$$

and therefore, the correctional functional for solving equation (19) using case 2 with the VIM:

$$
\begin{aligned}
\underline{y}_{n+1}(x, \alpha)= & \underline{y}_{n}(x, \alpha)+\int_{0}^{x}\left(e^{s-x}-1\right)\left[\underline{y}_{n}^{\prime \prime}(s, \alpha)-\right. \\
& \left.4 \bar{y}_{n}^{\prime}(s, \alpha)+4 \underline{y}_{n}(s, \alpha)\right] \mathrm{d} s \\
\bar{y}_{n+1}(x, \alpha)= & \bar{y}_{n}(x, \alpha)+\int_{0}^{x}\left(e^{s-x}-1\right)\left[\bar{y}_{n}^{\prime \prime}(s, \alpha)-\right. \\
& \left.4 \underline{y}_{n}^{\prime}(s, \alpha)+4 \bar{y}_{n}(s, \alpha)\right] \mathrm{d} s
\end{aligned}
$$

where $n=0,1, \ldots$. Hence, to find $\underline{y}_{1}$ and $\bar{y}_{1}$, we choose the initial approximate solutions for the VIM as:

$$
\begin{aligned}
& \underline{y}_{0}(x, \alpha)=(2+\alpha)+(5+\alpha) x \\
& \bar{y}_{0}(x, \alpha)=(4-\alpha)+(7-\alpha) x
\end{aligned}
$$

which implies respectively upon applying equations (20) and (21) to:

$$
\begin{aligned}
\underline{y}_{1}(x, \alpha)= & (2+\alpha)+(5+\alpha) x+(8 \alpha-20)+(20+4 \alpha) \\
& (x-1)-(8 \alpha-20) x-(20+4 \alpha) \frac{x^{2}}{2}-(8 \alpha \\
& -20) e^{-x}+(20+4 \alpha) e^{-x} \\
\bar{y}_{1}(x, \alpha)= & (4-\alpha)+(7-\alpha) x+(28-4 \alpha)(x-1)+ \\
& (4+8 \alpha) x-2 x^{2}(7-\alpha)+(4+8 \alpha) e^{-x}+ \\
& (28-4 \alpha) e^{-x}
\end{aligned}
$$

Sketch of the results for the lower and upper solutions with different levels $\alpha=0.2,0.4,0.6,0.8$ and 1 are given in Figures 1 and 2, respectively for the proposed method and that method followed in [3]. 


\section{Al-Nahrain Journal of Science}

ANJS, Vol.24 (4), December, 2021, pp. 32-39

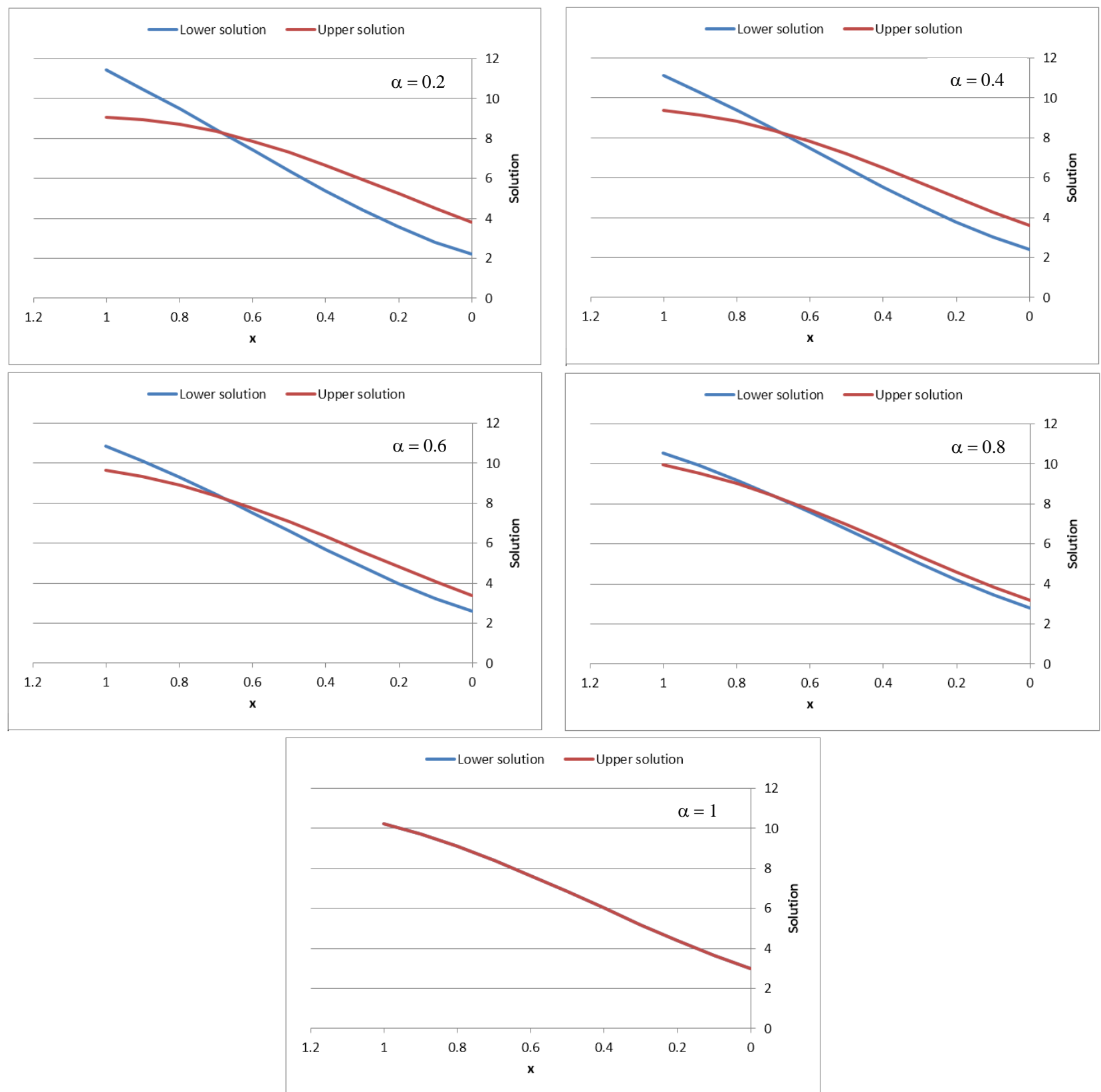

Figure 1. One iteration lower and upper solutions of Example 2 using the modified VIM for different values of $\alpha$-levels. 


\section{Al-Nahrain Journal of Science}

ANJS, Vol.24 (4), December, 2021, pp. 32-39

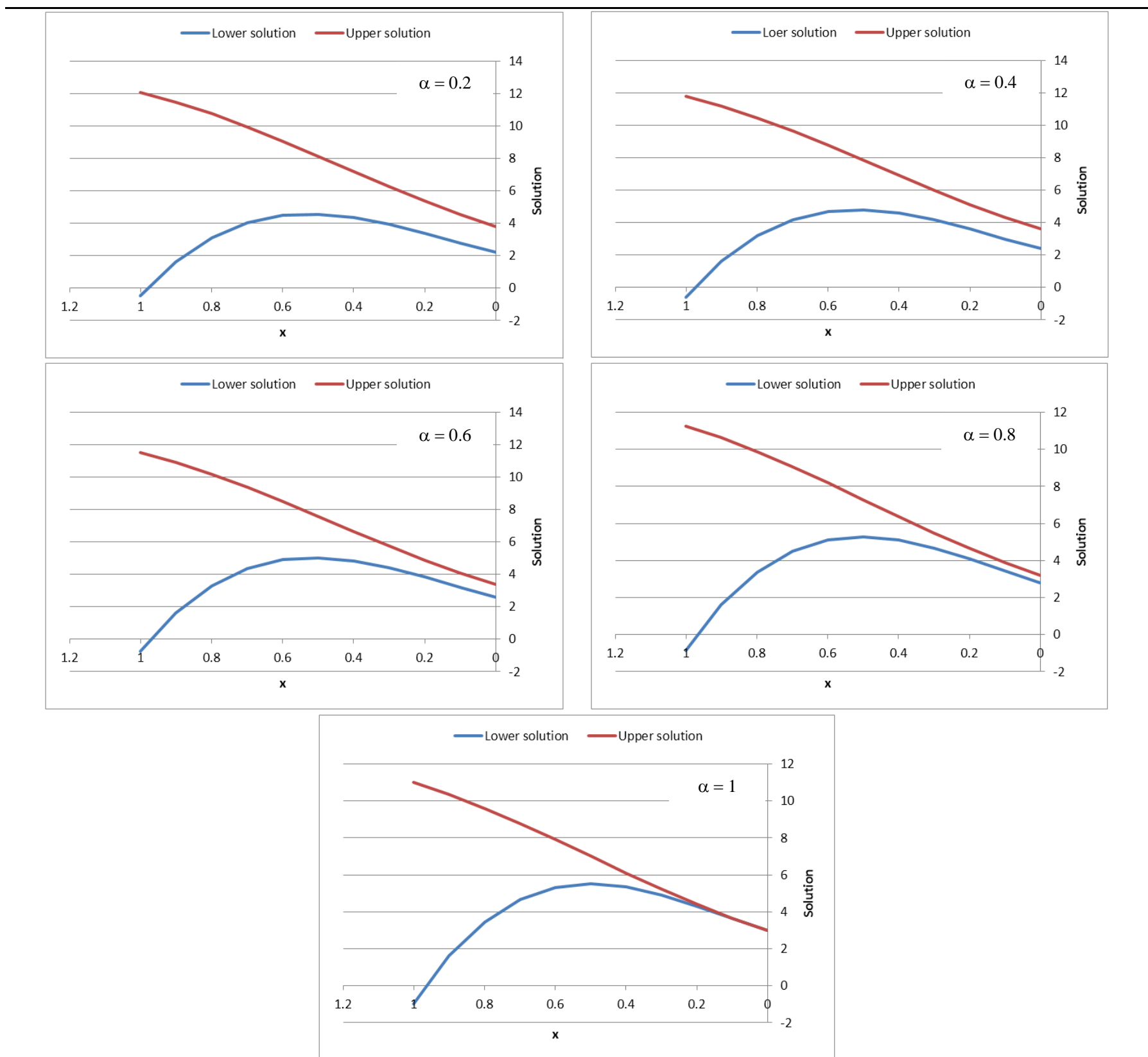

Figure 2. Three iterations lower and upper solutions evaluated by Jafari H. et al. [3] of Example 2 for different values of $\alpha$ levels.

\section{Conclusions}

A modified approach of the VIM is proposed in this paper which is applied successfully to find the solution of the $n$-th order linear FODE. A very high accurate results are obtained using only one iteration and thus the rate of convergence is highly increased, as expected which is due to the new value of the general Lagrange multiplier. Also, as it is seen from the results of Example 2 that the results obtained in [3] extremely wrong, as it is checked for $\alpha=1$, which is unequal to the crisp solution.

\section{References}

[1] Zadeh L. A., "Fuzzy sets". Information and Control, 8: 338-353, 1965.

[2] Abbasbandy S. and Viranloo T. A., "Numerical solutions of fuzzy differential equations by Taylor method". Computational Methods in Applied Mathematics, 2(2): 113-124, 2002.

[3] Jafari H., Saeidy M. and Baleanu D., "The variational iteration method for solving n-th order fuzzy differential equations". Central European Journal of Physics, 10(1): 76-85, 2012.

[4] Allahviranloo T., Abbasbandy S. and Behzadi S. S., "Solving nonlinear fuzzy differential equations by using 


\section{Al-Nahrain Journal of Science}

ANJS, Vol.24 (4), December, 2021, pp. 32-39

\begin{abstract}
fuzzy variational iteration method". Soft Computing, 18(11): 2191-2200, 2014.
\end{abstract}

[5] Jameel A., "The variational iteration method for solving fuzzy Duffing's equation". Journal of Interpolation and Approximation in Scientific Computing, 2014(2014): 1$14,2014$.

[6] Guo X. and Shang D., "Approximate solution of thorder fuzzy linear differential equations". Mathematical Problems in Engineering, 2013, 2013.

[7] Ghazanfari B. and Sepahvandzadeh A., "Variational iteration method for solving hybrid fuzzy differential equations". Journal of Mathematical Extension, 10: 75$85,2016$.

[8] Narayanan S. L., "An Adomian decomposition method to solve fuzzy Cauchy nonlinear differential equations". International Journal of Scientific Research and Modern Education (IJSRME), 2(1): 91-97, 2017.

[9] Narayanamoorthy S. and Yookesh T., "Variational iteration method to solve linear fuzzy delay differential equations using He's polynomials", 2017.

[10] Behzadi S. S., "The use of fuzzy variational iteration method for solving second-order fuzzy Abel-Volterra integro-differential equations". International Journal of Industrial Mathematics, 10(2): 211-219, 2018.

[11] Abbasbandi S. and Jafarian A., "Fixed point method for solving fuzzy nonlinear equations". Journal of Sciences (Islamic Azad University), 18: 2009.

[12] Farajzadeh A., Pour A. H. and Amini M., "An explicit method for solving fuzzy partial differential equation". International Mathematical Forum, 5(21): 1025-1036, 2010.

[13] Tapaswini S. and Chakraverty S., "New analytical method for solving n-th order fuzzy differential equations". Ann. Fuzzy Math. Inform., 8: 231-244, 2014.

[14] Tapaswini S., Chakraverty S. and Allahviranloo T., "A new approach to $n$-th order fuzzy differential equations". Computational Mathematics and Modeling, 28(2): 278-300, 2017.

[15] Jafari H. and Alipoor A., "A new method for calculating general Lagrange multiplier in the variational iteration method". Numerical Methods for Partial Differential Equations, 27(4): 996-1001, 2011.

[16] Wazwaz A. M., "The variational iteration method for solving linear and nonlinear Volterra integral and integro-differential equations". International Journal of Computer Mathematics, 87(5): 1131-1141, 2010.

[17] Narayanamoorthy S. and Mathankumar S., "Variational iterative method: an appropriate numerical scheme for solving system of linear Volterra fuzzy integro-differential equations". Advances in Difference Equations, 2018(1): 1-15, 2018.

[18] Jafari R. and Razvarz S., "Solution of fuzzy differential equations using fuzzy Sumudu transforms". Mathematical and Computational Applications, 23(1): 5, 2018.
[19] Hasan1 N. N. and Fadhel F. S., "Variational iteration method for solving system of fractional order ordinary differential equations". IOSR Journal of Mathematics, 10(6): 48-54, 2014. 